\title{
A Case Study on Antibiotic Loaded Calcium Sulphate Beads in the Management of Diabetic Foot Complications
}

\author{
Rajesh Kesavan", Changam Sheela Sasikumar and Vimalkumar Ramachandran
}

Diabetic Foot Specialist, Podiatric Surgeon, Hycare for Wounds (A Unit of NRA Advanced Wound Care Pvt Ltd), India

\begin{abstract}
*Corresponding author: Dr. Rajesh Kesavan, Diabetic Foot Specialist, Podiatric Surgeon, Hycare for Wounds (A Unit of NRA Advanced Wound Care Pvt Ltd), \#91-A, Hussain Complex, Millers Road, Kilpauk, Chennai, Tamilnadu, India, E-mail: hycareforwound@gmail.com
\end{abstract}

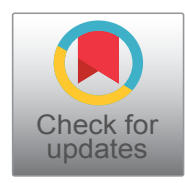

\section{Introduction}

Foot infections are the most frequent cause for hospitalization and the immediate forerunner to lower-extremity amputation in Diabetes [1]. Infection usually starts in ulcerated soft tissues but can spread contiguously to underlying bone [2] leading to osteomyelitis, which can affect any bone but most frequently the forefoot $(90 \%)$, followed by the midfoot $(5 \%)$ and the hind foot (5\%). Forefoot have a better prognosis than midfoot and hind foot osteomyelitis [3]. Despite the variety of available treatment options, including surgical procedures and antimicrobial therapy, bone infections are still a challenge to the professionals. In this case report we demonstrated the application of a synthetic, biodegradable and biocompatible form of calcium sulphate as a drug delivery system to treat a diabetic foot ulcer complicated by osteomyelitis.

\section{Case Report}

A 62-year-old Female patient with Type 2 diabetes ( $\mathrm{Hb} \mathrm{A} 1 \mathrm{C}>7)$ of 12 years duration with controlled hypertension and was referred to our Hycare Wound Care Centre for immediate assessment due to a limb threatening foot infection. The diabetologist had counselled the patient with regards to the likelihood of amputation. Local examination revealed tenderness with increased local temperature, swelling and erythema, with complaints of pyrexia, pain on the left fore foot and restricted movement of the left leg. Neurological testing demonstrated profound peripheral neuropathy with no ability to appreciate a $10 \mathrm{~g}$ monofilament or vibration sensation within the foot. Radiograph demon- strated the presence of osteomyelitis at the hallux (Figure 1) in the left fore foot. Patient underwent surgery by means of an incision and drainage procedure with local antibiotic administration to augment systemic antibiotics. HPS calcium sulfate powder was mixed with $1 \mathrm{~g}$ of vancomycin hydrochloride and $80 \mathrm{mg}$ of gentamicin sulfate in the form of pellets. Vancomycin and gentamicin were selected as the antibiotics based on in vitro sensitivities of isolates obtained from diabetic foot infections. Sequestrate were excised, the bone was fenestrated and packed with the pellets, and primary closure was carried out. The beads were packed into the wound and around the remaining bone to ensure thorough coverage within the foot. The skin edges were apposed with single non-absorbable interrupted sutures and dressed appropriately. The ulcer was defined as fully healed when epithelization was complete, and the intervention was considered successful when there was no reoccurrence for 12 months.

\section{Discussion}

Osteomyelitis is challenging to treat due to multidrug resistance of common pathogens and poor penetration of antibiotics into bone [4]. Hemihydrate form of Calcium Sulfate is produced using a synthetic process resulting in pure grade with no traces of toxic impurities. It has the advantage of delivering a wider spectrum of antibiotic combinations into the affected joint.

Osteomyelitis is challenging to treat due to both multidrug resistance microbes and to poor penetration of antibiotics into the bone. Inadequate release of antibiotics to the site of infected bone is a frequent problem

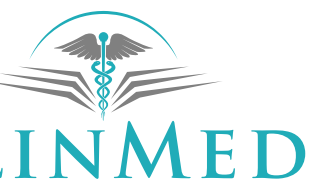

INTERNATIONAL LIBRARY 


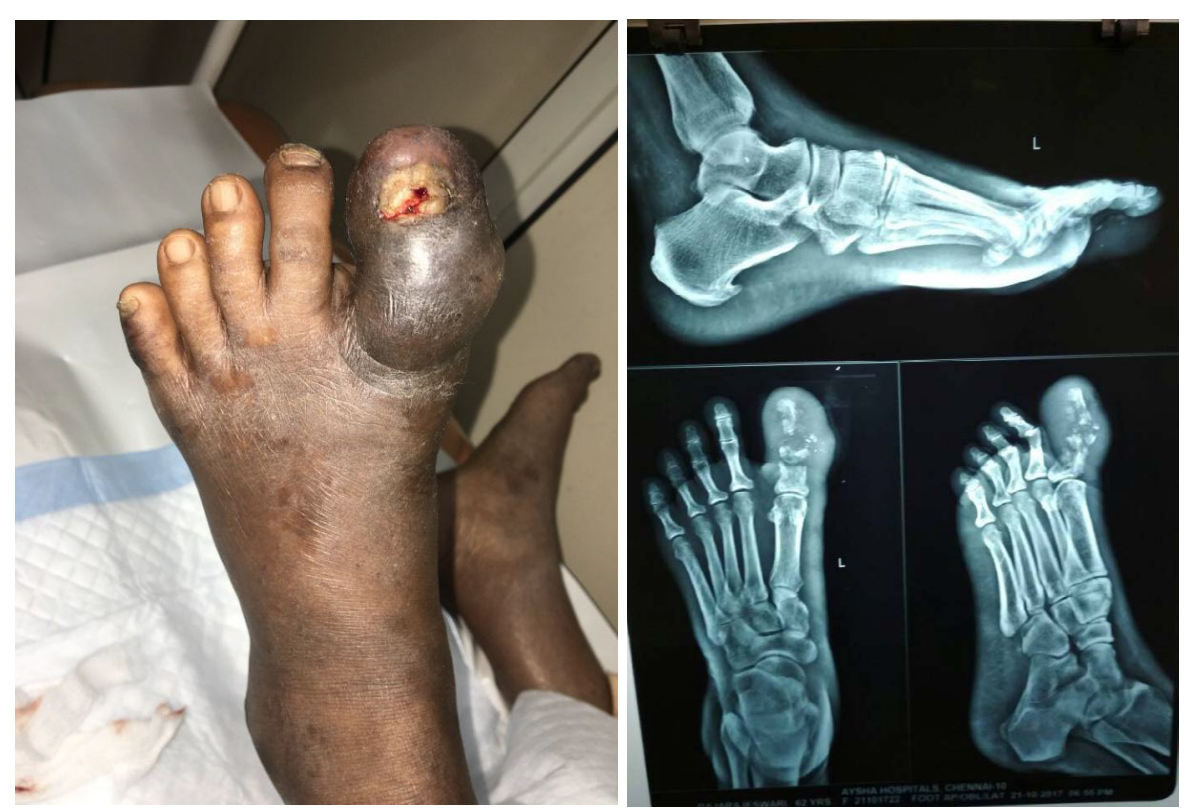

Figure 1: Foot $x$-ray showing osteomyelitis at the hallux.

AFTER 2 WEEKS

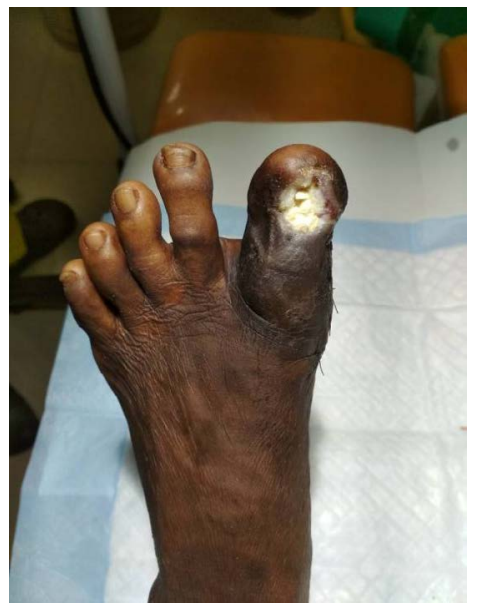

FIG: 2 A

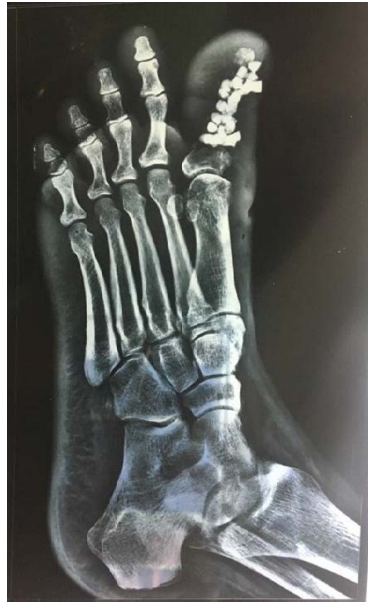

FIG: 2 B
AFTER 4 WEEKS

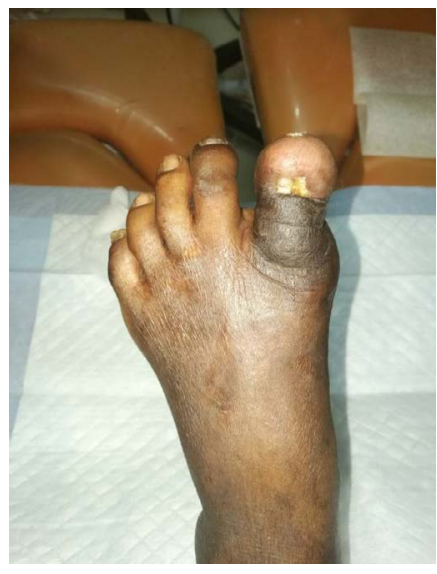

FIG: 2 C

Figure 2: Post-operative x-ray showing partially absorbed calcium sulfate beads.

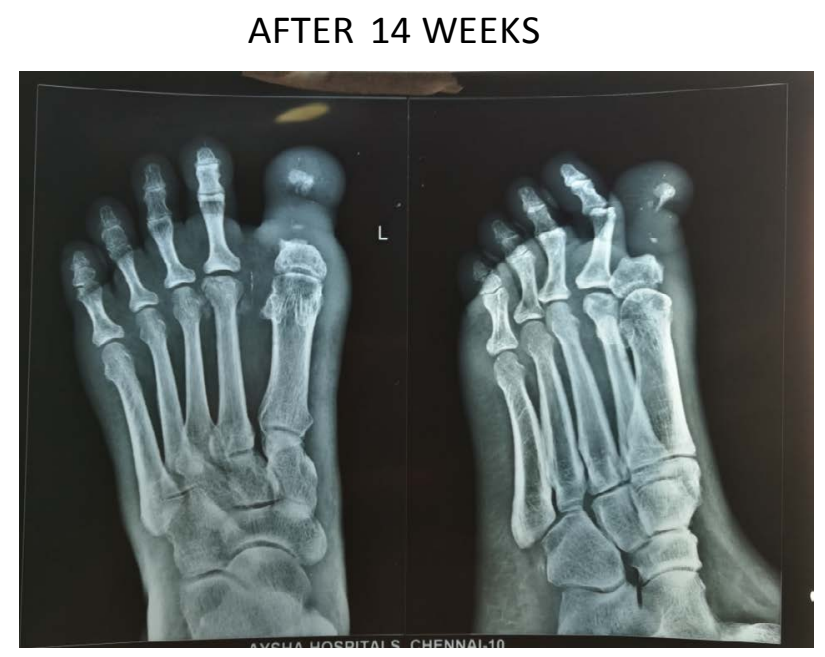

FIG: 3 A

\section{AFTER 14 WEEKS}

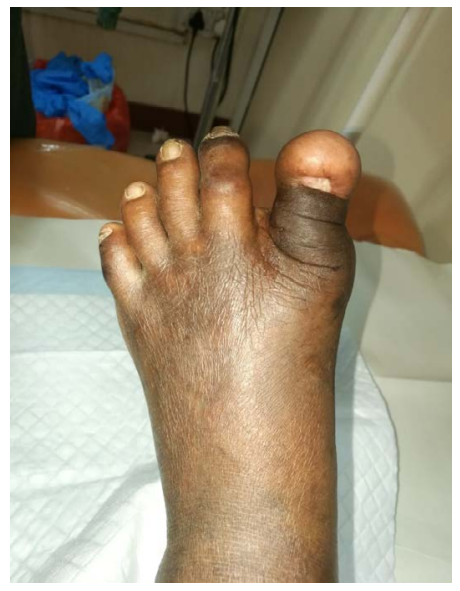

FIG: 3 B

Figure 3: Post-operative $x$-ray completely absorbed calcium sulfate beads. 
associated with systemic antibiotic therapy and sometimes even with local drug delivery system. Purified synthetic calcium sulphate is absorbable, can be readily mixed with antibiotics and positioned at the site of infection. This technique provides advantages over other local antibiotic delivery systems as it is biodegradable, predicable elution characteristics, osteoconductive and finally it can fill the dead space.

Post-Operative Radiograph evaluation was performed (Figure 2A and Figure 2B) in the patient after 2 weeks and later at 4 weeks to find out the bony integration of the calcium sulphate impregnated with antibiotic beads. Patient achieved healing with a median time span of 4 weeks and no recurrence within 12 months after intervention (Figure 2C). The need for postoperative antibiotics was decided as per clinical assessment and here the patient was prescribed with Ceftriaxone $(500 \mathrm{mg}) /$ day for a period of 5 days. Foot X-ray of the patient after 14 weeks is presented in Figure 3A and Figure 3B.

This procedure is found to be safe and effective for the treatment of forefoot diabetic osteomyelitis. No adverse reaction was noted in the patient and now with confidence our centre offers this treatment to the patients with midfoot and calcaneal osteomyelitis.

Our ultimate aim is to eradicate infection, heal the ulceration and reduce the need for intravenous antibiotics in the treatment of osteomyelitis. Hence this protocol was adopted to achieve an alternative route of administration of antibiotics in the management of Diabetic foot ulcers.

\section{References}

1. Farhang Babamahmoodi, Tahereh Shokohi, Fatemeh Ahangarkani, Mojtaba Nabili, Elham Afzalian, et al. (2015) Rare case of aspergillus ochraceus osteomyelitis of calcaneus bone in a patient with diabetic foot ulcers. Case Reports in Medicine 2015: 509827.

2. EM Shankar, V Mohan, G Premalatha, RS Srinivasan, AR Usha (2005) Bacterial etiology of diabetic foot infections in South India. Eur J Intern Med 16: 567-570.

3. Laura Giurato, Marco Meloni, Valentina Izzo, Luigi Uccioli (2017) Osteomyelitis in diabetic foot: A comprehensive overview. World J Diabetes 8: 135-142.

4. Ibrahim E Abdellatif (2014) Local application of calcium sulphate impregnated with vancomycin and tobramycin in the treatment of chronic osteomyelitis. AAMJ 12: 96-109. 